\title{
Should We Postpone Elective Cardiovascular Procedures and Percutaneous Coronary Interventions During the COVID-19 Pandemic?
}

\author{
Gokhan Keskin, MD, ${ }^{1}$ Emced Khalil, MD, ${ }^{2}$ Ayhan Uysal, MD $^{3}$ \\ ${ }^{1}$ Department of Cardiology, Amasya University School of Medicine, Research and Education Hospital, Amasya, Turkey; ${ }^{2}$ Department \\ of Cardiovascular Surgery, Ordu University School of Medicine, Research and Education Hospital, Ordu, Turkey; ${ }^{3}$ Department of \\ Cardiovascular Surgery, Firat University School of Medicine, Elazı, Turkey
}

\section{ABSTRACT}

Background: Prioritization among patients with coronary artery disease represents a difficult issue during the SARS-CoV-2 pandemic. We present our clinical practices and patients' outcomes after elective, emergent and urgent cardiovascular surgery and percutaneous coronary interventions (PCI). We also investigated the rate of nasocomial infection of SARS-CoV-2 in health workers (HWs), including surgeons after cardiovascular procedures and percutaneous interventions (PCI).

Material and methods: We performed 186 cardiovascular operations and PCI between March 15 and October 15. According to level of priority $(\mathrm{LoP})$, we performed urgent and emergent coronary artery bypass grafting (CABG) and cardiac valve repair or replacement surgery in 44 patients. In one patient with acute chordae rupture with pulmonary edema, we performed mitral valve replacement. We performed aortic arch repair in two patients with type-I aortic dissection in urgent situations. Therefore, in 47 patients we performed cardiac operations in urgent or emergent situations. Elective CABG $(\mathrm{N}=28)$ and elective cardiac valve $(\mathrm{N}=10)$ surgeries were performed (total: 38$)$. While rescue PCI were urgently performed in 47 patients with ST segment elevation myocardial infarction (STEMI), it was performed in elective or emergent situations in 40 patients with myocardial ischemia. Endovascular treatment was performed in four patients with deep venous thrombosis (DVT) and in four patients with chronic arterial occlusion, respectively. Surgical vascular repair and embolectomy were performed in patients with peripheral artery injury $(\mathrm{N}$ $=6$ ) and acute arterial embolic events $(\mathrm{N}=4)$, respectively. We performed thoracic computed tomography followed by reverse transcriptase-polymerase chain reaction (RT-PCR) test in patients with irregular diffuse reticular opacities with or without consolidation on chest X-ray. Blood coagulation disorders including d-dimer, thromboplastin time (TT),

Received October 14, 2020; accepted November 1, 2020.

Correspondence: Gokban Keskin, Amasya University School of Medicine, Department of Cardiology, Akbilek Street. National Domination Campus, No:4/3, 05100 Merkez/Turkey (e-mail:keskinand@gmail.com. and partial thromboplastin time (aPTT) were measured prior to procedures.

Results: No mortality and morbidity was seen after percutaneous and surgical arterial or venous procedures. Total mortality rate was $4.1 \%$ (8 of $186 \mathrm{CAD}$ patients or valve surgery) after urgent and emergent $\mathrm{CABG}(\mathrm{N}=4)$, an urgent valve replacement $(\mathrm{N}=1)$, and PCI $(\mathrm{N}=3)$. Low cardiac output syndrome (LOS) and major adverse cardiac cerebrovascular event (MACCE) were the mortality factors after cardiac surgery. The reasons for death after PCI were sudden cardiac arrest related to dissection of the left main coronary artery during procedure and pneumonia due to COVID-19 $(\mathrm{N}=2)$. Ground-glass opacities in combination with pulmonary consolidations was detected in seven patients. Interlobular septal and pleural thickening with patchy bronchiectasis in the bilateral lower lobe involvement was found after thoracic computed tomography in these patients. We confirmed in-hospital COVID-19 using PCR test in two patients with STEMI prior to PCI. PT and aPTT increased, but fibrin degradation products did not in those two patients. We confirmed COVID-19 via phone call in six CABG patients and one PCI patient after discharge from hospital. None of the patients diagnosed with COVID-19 died after being discharged from the hospital.

Conclusion: Cardiovascular surgery and PCI can safely be performed with acceptable complications and mortality rates in elective situations, during the COVID-19 pandemic. Preoperative control of OR traffic, careful evaluation of the patient's history, consultation, and precautions taken by healthcare professionals are important, during and after procedures. Also important is the wearing a mask and face shield and careful disinfection of equipment and space.

\section{INTRODUCTION}

The first case with COVID-19, or SARS-CoV-2, was reported in Wuhan, China in 2019. Since many countries reported surging numbers of patients, The World Health Organization (WHO) accepted the spread of COVID-19 as a pandemic in March 2020. This virus is a cause of acute respiratory distress syndrome (ARDS) with or without an intravascular hypercoagulation state especially in risky patients, such as those with coronary artery disease (CAD), 
Table 1. Characteristics of $\mathrm{PCl}$ patients

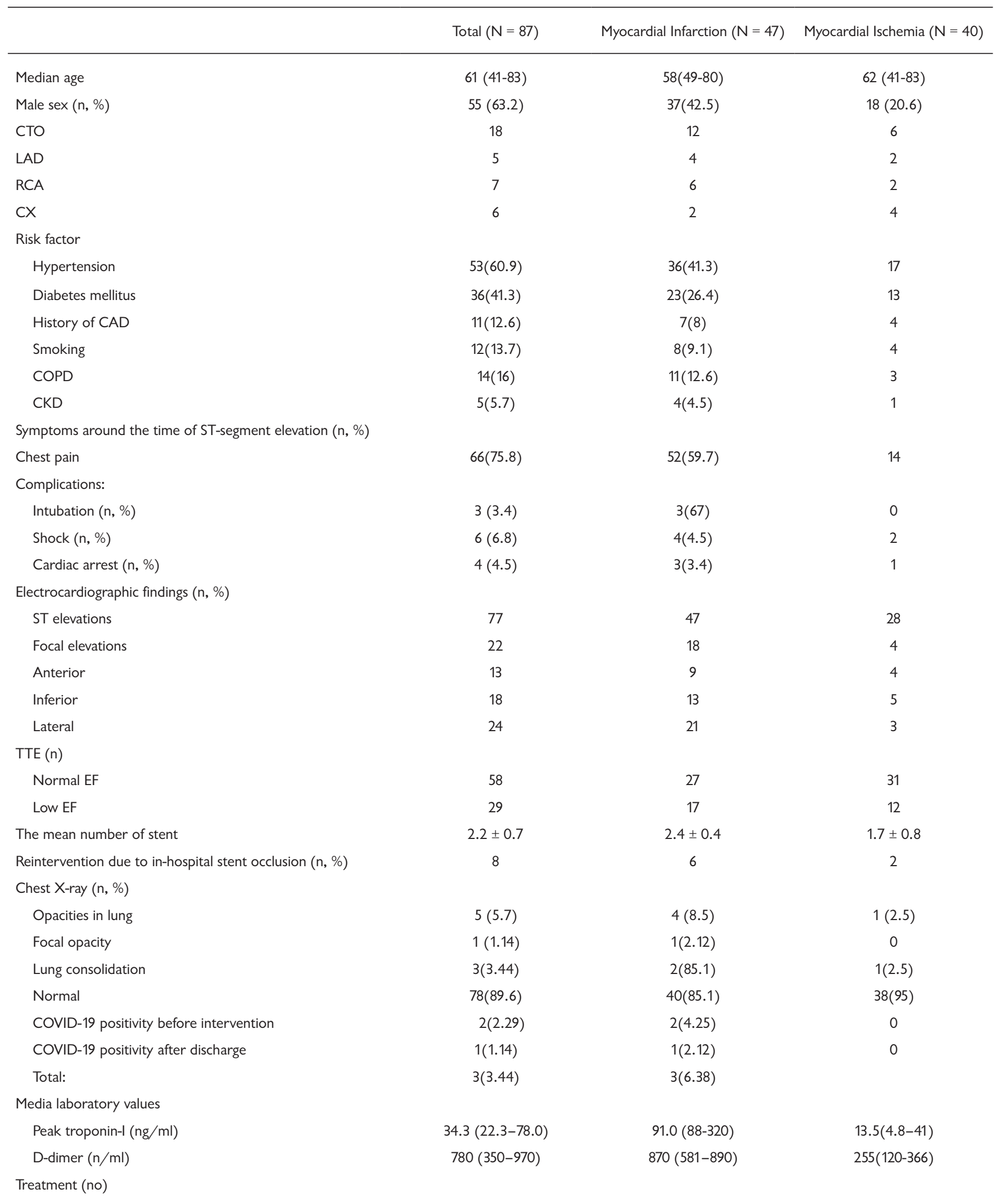


Table 1. Characteristics of $\mathrm{PCl}$ patients [Cont.]

\begin{tabular}{lccc}
\hline & Total $(\mathrm{N}=87)$ & Myocardial Infarction $(\mathrm{N}=47)$ & Myocardial Ischemia $(\mathrm{N}=40)$ \\
\hline Fibrinolytic agent & 6 & 4 & 2 \\
Statins & 81 & 65 & 16 \\
ACE receptor blocker & 87 & 47 & 40 \\
Beta-blocker & 87 & 47 & 40 \\
Antihypertensive & 53 & 33 & 20 \\
Aspirin & 66 & 39 & 27 \\
Clopidogrel & 87 & 47 & 40 \\
Death in-hospital $(n, \%)$ & $3(3.44)$ & $3(6.3)$ & 0 \\
\hline
\end{tabular}

The reference value for the troponin-I and the $\mathrm{d}$-dimer were $0.06 \mathrm{ng} / \mathrm{mL}$, and $230 \mathrm{ng}$ per milliliter or less, respectively.

hypertension, diabetes, older age with some comorbidities, etc. [Callaway 2020; Cheung 2000; WHO 2005; Liu 2020; Turner 2004]. About half of in-hospitalized patients diagnosed with COVID-19 had some comorbidities [Mallapaty 2020; Chen 2020; Poon 2020; Chan 2020; Wang 2020; Huang 2020; Zheng 2020]. Because the spread of SARS-CoV-2 follows an exponential curve, management of cardiovascular disease includes some controversy.

To decrease mortality and morbidity of patients with severe CAD requiring revascularization and professional HWs nasocomial infection of COVID-19, The World Health Organization (WHO) has accepted that this severe situation has become a pandemic [WHO 2020]. The American College of Surgeons [WHO 2020] and American Thoracic Surgery [American College of Surgeons 2020] suggest postponing elective cardiovascular surgery. Also, about the half of CAD patients have hypertension and/or diabetes mellitus. Therefore, revascularization procedures are very important in these patients. In addition, postponing cardiovascular surgery or PCI restricts daily activities of these patients and may increase the risk of sudden death related to myocardial infarction or another cardiovascular complication, such as MACCE. In addition, some authors suggest that COVID19 may be the cause of myocardial injury, severe arrhythmia, acute coronary syndrome (ACS), and thromboembolic events as a first symptom [Huang 2020; Zheng 2020; WHO 2020; American College of Surgeons 2020; WHO 2020]. Thus, the main question is: What is the risk/benefit ratio regarding the decision to postpone or proceed with elective cardiovascular surgery or percutaneous interventions during the SARS$\mathrm{CoV}-2$ pandemic?

At the beginning of the COVID-19 pandemic, there were no clear algorithms with regard to cardiovascular surgery or percutaneous interventions to provide nasocomial transmission of COVID-19, and no suggestions for preparing surgical equipment, use of technical devices, or control of OR traffic or the Cath. Lab. As a result, both cardiologists and surgeons had some problems regarding the precautions to be taken with patients and HWs with or without risk factors in revascularization procedures [Aguilar 2020]. After suggestions by international scientific committees and the WHO about patient prioritization, we performed surgery and PCI, according to level of priority (LoP). Elective cases are defined as LoP I that may not be postponed. We reconsider LoP II to IV cases, according to the team of cardiologists/cardiac surgeons. During the COVID-19 pandemic, the number of patients with acute coronary syndrome admitted to our hospital decreased by approximately $40-45 \%$. Herein, we present the outcomes of our cardiovascular surgical procedures, and PCI during eight months with a limited number of patients. We discuss the risks/benefits ratio regarding the decision whether to postpone or continue with elective coronary revascularization and vascular procedures during the COVID-19 pandemic, considering the specific risks of these cardiac patients.

\section{MATERIAL AND METHODS}

Hemoglobin and hematocrit levels, white blood cell, and thrombocyte counts routinely were measured. Electrocardiography, transthoracic echocardiography, cardiac enzyme levels (troponin-I and CKMB) were measured at admission to the emergency department. If the patient had typical angina pectoris with electrocardiographic changes, tachypnea or dyspnea with or without tachycardia-bradycardia syndrome, respiratory function tests were investigated prior to transportation to the ICU. To prevent in-hospital spread of COVID19 via nosocomial transmission for both patients and professional HWs, including the anaesthetist, surgeons, and cardiologist, precautions were taken. One OR separated the suspected or confirmed COVID-19 patients, who required cardiovascular surgery and interventions. The cardiac team performed the triage of patients with great care after consultation, which included the emergency unit of the hospital.

We performed elective $(\mathrm{N}=34)$, emergent $(\mathrm{N}=10)$, and urgent $(\mathrm{N}=28)$ cardiac operations. Arterial or venous percutenous interventions and surgeries were performed in 18 patients, including acute vascular injuries. Ten vascular surgeries, including vascular injuries or embolic events, were 
Table 2. Characteristics of the patients undergoing cardiovascular surgery

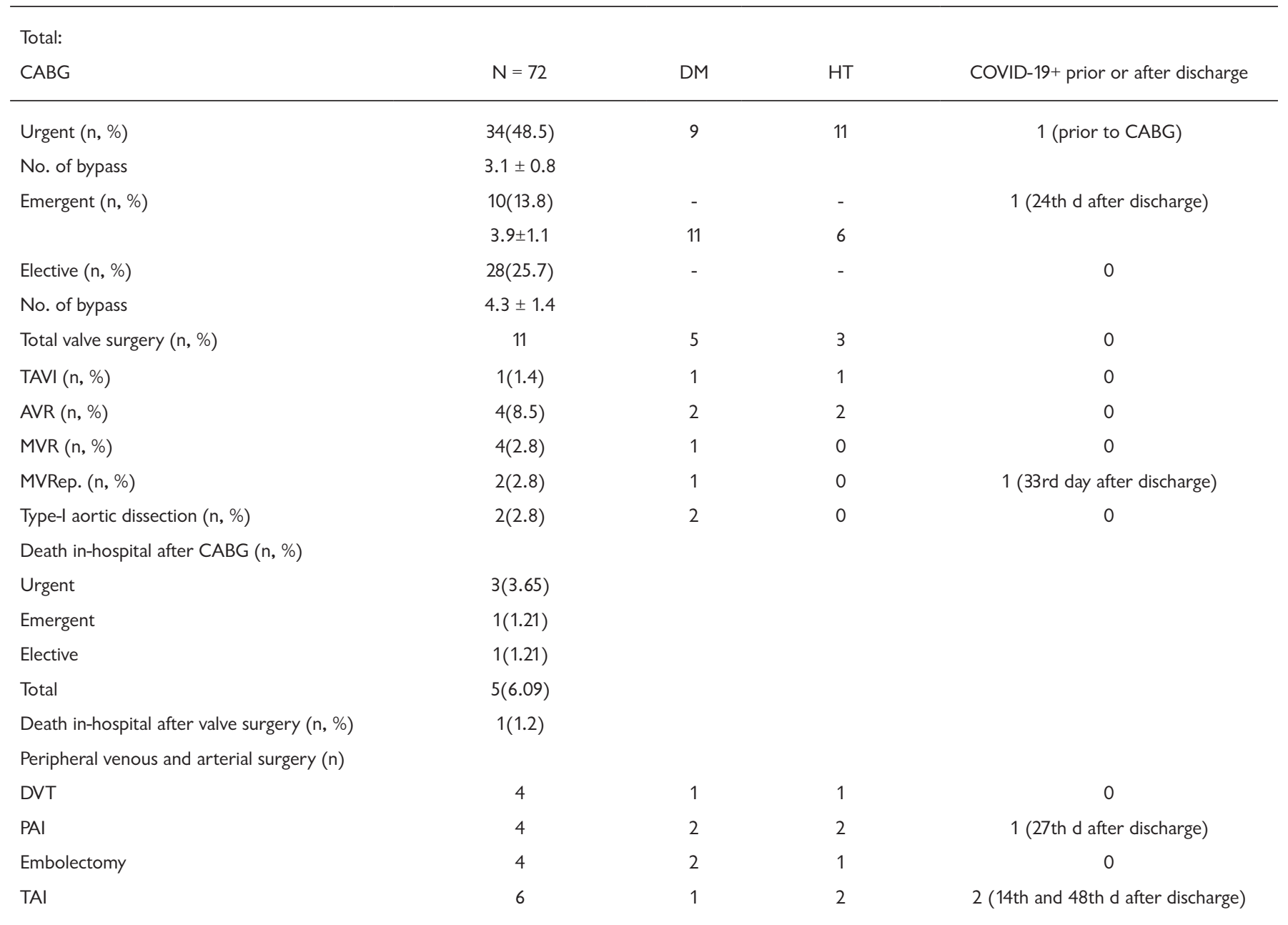

CABG: coronary artery bypass grafting; TAVI: transcatheter aortic valve inserion; AVR: aortic valve replacemen; MVR: mitral valve replacemen; MVRep; mitral valve repair; DVT: deep venous thrombosis; PAO: peripheral artery intervention; TAl: traumatic arterial injury; HT: hypertension; DM: diabetes mellitus

performed in emergent situations. Overall, the male/female ratio was $102 / 84$. The median age was 56 years. The characteristics of PCI, CABG, valvular operations, vascular surgeries, and vascular interventions are summarized in Tables 1 and 2.

For each patient, we performed a questionnaire that consisted of relevant signs and symptoms of COVID-19, such as fever, cough, shortness of breath or difficulty breathing, loss of smell, and upper airway congestion. We also investigated patient history and whether there was contact with another COVID-19 patient or if the patient had traveled from another country. We took nasopharyngeal swabs for PCR tests in 17 suspected patients because of the history of fever, nasal congestion with or without loss of smell, and dysnea or tachypnea. In addition, PT, aPTT, fibrin degradation, and d-dimer were measured to explain hypercoagulation. We confirmed COVID-19 in two CAD patients, who required urgent PCI. We consulted an infectious disease specialist with these patients.

We classified the patients ( $\mathrm{LoP}$ ), according to previous national and international societies' suggestions [Aguilar 2020] (Figure 1). We consulted the Association of European Thoracic and Cardiovascular Surgery, preparing OR or Cath. Lab. equipment prior to procedures and utilized surgical gloves, face masks (N95), and face shields. We mapped out OR traffic for personnel and patient protection. (Figure 2) We performed urgent and emergent CABG in 47 patients, including Type-I aortic dissection and an acute severe mitral failure.

Standard aortic and venous cannulation was performed, and extracorporeal circulation was used. Cardiac arrest was provided using an antegrade and retrograde cold blood cardioplegic solution after aortic cross-clamping. In three urgent patients, beating heart CABG surgery was performed. Elective CABG operations were performed in 28 patients. The mean number of revascularized coronary vessels was 3.8 


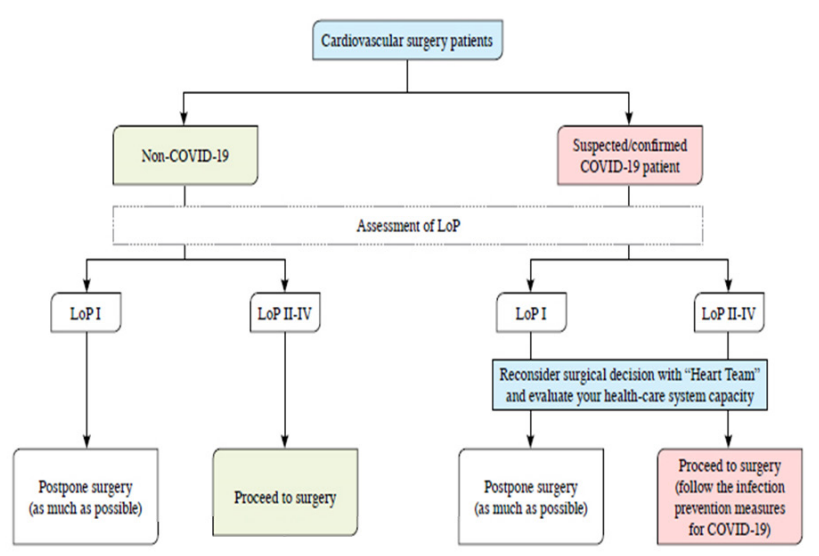

Figure 1. The algorithm of cardiovascular surgery. According to our national protocols, we classified the patients requiring coronary artery bypass grafting.

\pm 1.1 (range: $1-5$ ). Mitral valve replacement or repair were performed in six patients, and aortic valve replacement electively was performed in four patients. The left and the right internal thoracic artery, radial artery, and saphenous vein were used as bypass conduits. The mean operation time and ECC time for open heart surgery were $244 \pm 56.4$ minutes and $97 \pm 16.4$ minutes, respectively.

During the preoperative period, we confirmed COVID-19 in our two patients requiring CABG. Therefore, we consulted an infectious disease specialist. After 14 days of treatment, the PCR test was negative. Thus, we operated on the patient at the end of the 21 st day. We performed PCI for another patient. Unfortunately, he died on the fifth day due to acute stent thrombosis.

There were different levels of patchy or reticular opacities and consolidation of chest X-ray in seven patients (Figure $3 \mathrm{~A}$ ). Thoracic computed tomography was performed in patients with COVID-19 symptoms although the COVID-19 PCR test was negative. Interlobular septal and pleural thickening with bilateral lower lobe congestion are important indications of COVID-19 (Figure 4). These patients underwent a second PCR test. To determine whether COVID-19 treatment should be initiated or not for these patients, we consulted an infectious disease specialist and chest disease specialist. We also repeated the measurement of blood levels of PT, aPTT, international normalized ratio (INR), and D-dimer in patients with COVID-19 or in patients with the symptoms of upper and/or lower airway systems. All were within normal limits, and fibrin degradation products did not increase, except four CAD patients given a second COVID-19 tested positive. Thus, we postponed the CABG $(\mathrm{N}=2)$ and PCI $(\mathrm{N}=2)$ for four weeks.

Deep venous thrombectomy (DVT) was performed in four female patients because of recurrent venous thrombosis. Endovascular treatment electively was performed in four patients with chronic peripheral artery disease. Surgical approaches were performed in patients with acute arterial embolic events, and 10 patients suffered peripheral

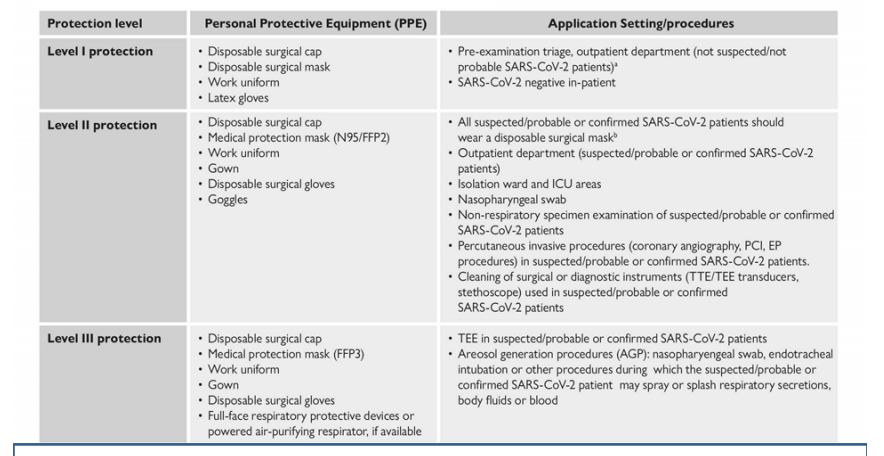

Figure 2. Personal protection and application setting for cardiovascular procedures, including level 1 to level III protection, that is proposed by international scientific committees. Disposable dress, gloves, and face masks $(\mathrm{N}-95)$ clearly are shown prior to treatment.

artery injury.

PCI protocols: Patients' characteristics and clinical results following treatment are summarized in Table 1. For confirmation of suspected COVID-19 patients, we used RT-PCR. There was bilateral lower lobe consolidation with pleural thickening and patchy or reticular opacities. Patients were isolated in a separate room, and we immediately performed stenting for culprit lesions.

Definition of coronary artery lesions: STEMI was described according to typical symptoms associated with STsegment elevation. Coronary artery stenosis was defined as the culprit lesion in patients with evidence of luminal occlusion or intracoronary thrombus during angiography. We described obstructive CAD based on the angiographic evidence of a stenosis $>50 \%$. We performed PCI in 87 patients with severe coronary artery stenosis and one TAVI procedure in a female who previously underwent mitral and tricuspid valvular replacement (Figure 5).

Male/female ratio was 55/32, and the mean age of 61 years. For PCI patients, the STEMI was the most common clinical manifestation. We confirmed COVID-19 test results in two patients with diabetes and hypertension at hospital admission. While urgent PCI were performed in 47 patients with STEMI, it was performed in $40 \mathrm{CAD}$ patients with myocardial ischemia in elective or emergent situations. Seventyeight percent of patients were referred to our hospital with angina pectoris associated with dyspnea, and 16 patients had dyspnea without chest pain (18.3\%). The success rate of PCI was $100 \%$.

On echocardiography, 33 patients $(37.9 \%)$ had localized wall motion abnormalities, 13 (14.9\%) had diffuse hypokinesia. The remaining patients did not have any ventricular abnormalities. The mean left ventricular ejection fraction was < 45 (range: $25-65 \%$ ) in 27 patients (31\%). We used fibrinolytic agents in six patients. Sixty-nine patients (79.3\%) had one or more culprit lesions. Chronic total occlusion was seen in 18 patients. As of March 11, 2020 (median follow up, 10 days; range, 3-17 days), three patients had died (3.4\%). Two of the three patients had COVID-19. Reinterventions successfully were performed in eight patients owing to acute 


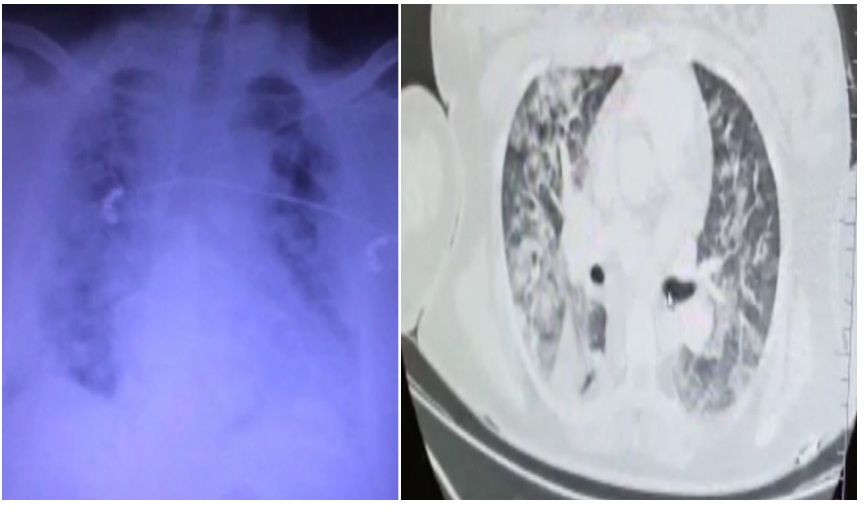

Figure 3. The pathologies of X-ray and thoracic computed tomography. There are reticular and patchy opacities on the X-ray (left side), and bilateral lung consolidation and pleural thickening, including bronchiectatic areas observed on the right side of a COVID-19 patient.

stent occlusion. The remaining patients were discharged to home. The main complications were shock, cardiac arrest, and intubation after PCI (Table 1).

During the SARS-CoV-2 outbreak, according to the WHO and scientific committee, the regional STEMI or myocardial ischemia network were reorganized in our Cath. Lab. and ICU. We have observed a significantly reduced (about 55\%) number of patients with STEMI referred to our hospital. The outbreak factor may be the main reason for the low number of patients during the study period. Our study findings showed that typical STEMI presentation may be performed to decrease mortality and morbidity owing to CAD in patients with a culprit lesion, except a type-I myocardial infarction.

\section{DISCUSSION}

The SARS-CoV-2 pandemic has brought many problems, such as the management of patients with severe cardiovascular diseases that require invasive or surgical intervention and professional HWs. The novel virus is more transmissible than other coronaviruses [Cheng 2019]. Angiotensin-converting enzyme 2 receptors and spike proteins of this virus infect human tissue through attachment, including lung, kidney, intestine and myocardium [Lei 2020]. Understanding the interaction between COVID-19 and cardiovascular procedures in $\mathrm{CAD}$ cases that require revascularization may help cardiovascular surgeons, anesthesists, and cardiologists decide whether to postpone or proceed with procedures during the pandemic. The American College of Surgeons and American Society of Anesthesiologysts suggest postponing elective surgeries and PCI because they may increase the mortality risk via nasocomial infection of patients and healthcare workers [Lei 2020].

Although a 14-day quarantine is recommended to prevent the spread of COVID-19 to healthy people during the pandemic period [Nepogodiev 2020], there may be an increase in deaths due to myocardial infarction in those with severe CAD

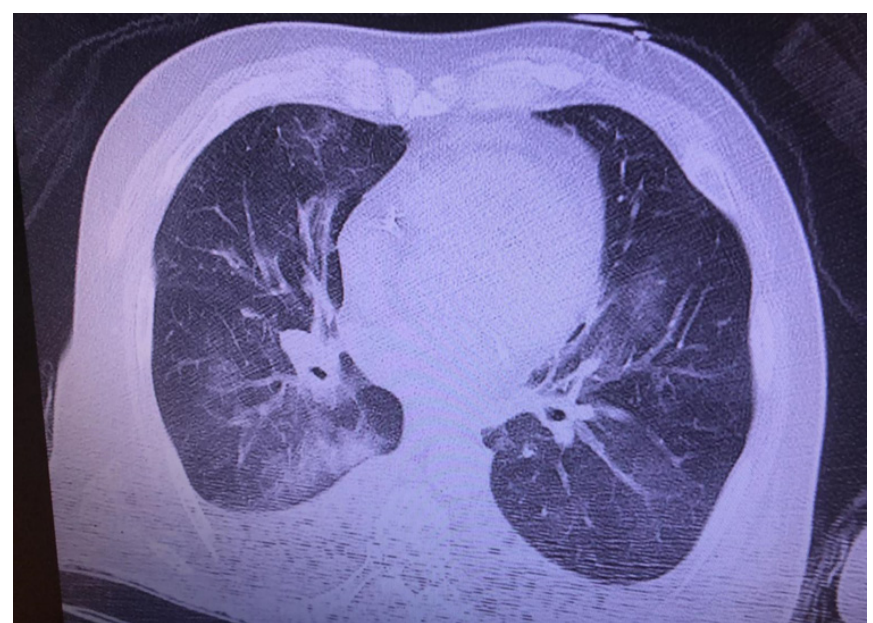

Figure 4. The pathologies of thoracic computed tomography. Bilateral lung consolidation is combined with edema and pleural thickening in this COVID-19 patient, who underwent percutaneous coronary intervention.

with hypertension and diabetes mellitus. On the other hand, delaying cardiovascular surgery or PCI can increase the risk the of complications resulting from the decrease the patient's activities. In our opinion, we have to discuss the risks/benefits ratio regarding whether to postpone or proceed with elective cardiac surgery and PCI, during the pandemic. As the virus has rapidly spread and high mortality rates have been reported from all countries, many surgical and interventional procedures have been postponed due to worldwide panic. Accordingly, sudden deaths at home increased.

Our study showed that elective cardiovascular surgery and PCI procedures may be performed with acceptable mortality and morbidity rates. According to suggested protocols, we prepared the OR and angiography unit, controlling the traffic in the ORs and Cath. Lab. Prior to surgery, special attention was made toward the sterilization of technical equipment; the patient also was isolated. To prevent nasocomial spread to healthcare professionals, we performed standard protocols [Fang 2020]. The clinical status of patients with COVID19-like symptoms, patient history including contact with a COVID-19 positive person, and travel from another country are important issues. We presented the previous suggestions of the OR, surgical instruments, and Cath. Lab. in Figure 2. We believe that performing revascularization of elective CAD patients may decrease death or complications from MACCEs related to cardiovascular events.

SARS-CoV-2 is highly contagious. Cardiac surgeons or cardiologists planning revascularization procedures are likely to encounter COVID-19 patients without symptoms. A clear understanding of the impact of this disease and its interaction with cardiovascular specialists will help cardiac surgeons and anesthesiologists properly decide whether to proceed with or postpone elective surgeries, during the current COVID19 pandemic [Chu 2020; Hernandez-Palaz 2018; Qiu 2020]. There are a number of points that cannot be clarified in determining the appropriate time for elective interventional procedures or open cardiac surgery. We think that early 


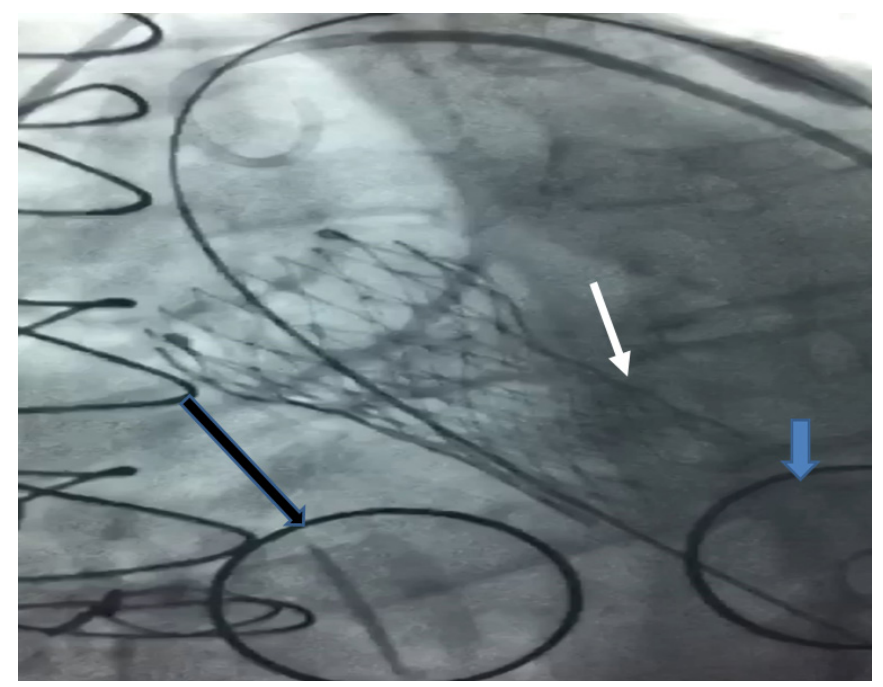

Figure 5. After transcatheter aortic valve replacement (white arrow) in a female patient who underwent previous mitral (blue arrow) and tricuspid valve replacement (black arrow). She had comorbidities, including obesity, hypertension, and diabetes mellitus.

intervention in coronary artery patients classified as elective cases with high risks for nosocomial infection during the pandemic will encourage surgeons and cardiologists.

We know that SARS-CoV-2 may trigger acute coronary syndromes in a small number of patients with COVID-19 referred with typical or atypical angina pectoris [Tully 2012; Dinan 2017; Coughlin 2012]. In 18 patients with COVID19 and ST segment elevation that showed acute myocardial infarction, five of six patients with myocardial infarction required PCI [Gaudriot 2015]. In other patients with COVID-19 referred with STEMI, coronary angiography demonstrated that 17 patients had evidence of a culprit lesion that required revascularization [Ruan 2020]. These authors suggested that STEMI might be the first clinical manifestation of COVID-19 for patients who had not yet tested positive upon hospital admission. All reports proposed that COVID-19 may be a cause of acute coronary syndrome even in the absence of classical symptoms or pulmonary complications due to SARS-CoV-2. Primary PCI remains the standard of treatment in patients with COVID-19 [Chen 2019; Bauer 2018; Huffmyer 2015; del Rio 2020; Bangalore 2020]. We believe that primary PCI should be performed for severely occluded coronary arteries, if we can provide a clinically safe Cath. Lab. In the event of a culprit lesion in another coronary artery, surgery may be postponed to minimize nasocomial spread of COVID-19. Mortality rate is high in patients with COVID-19 who underwent PCI; COVID-19 may precipitate a course of pneumonia or myocarditis, which is a main reason for admission to the ICU and increases mortality [Stefanini 2020].

We investigated COVID-19 positivity and related symptoms via calls to patients or their family members after hospital discharge. We were informed of positive COVID-19 results with six CABG patients and one PCI patient. All had minimal symptoms without ARDS or pneumonia related to COVID-19 after cardiac surgery or PCI. We performed thoracic computed tomography in a female who underwent CABG in the hospital. There was a slight pulmonary consolidation in this patient, and she was discharged to home on the fifth day. During the follow-up period (the mean time: $4.1 \pm$ 1.2 months; range: 22 d-6 months), no mortality or morbidity was seen.

Unfortunately, there was no correlation between laboratory findings, radiological investigation, clinical signs and symptoms, and the patient's history of contact with COVID19. While PCR and serological methods are used to diagnose COVID-19, false sampling, the inconsistency of samples and preparations still are the main problems that lead to false-negative results [Minamino-Muta 2017]. A retrospective study that included 34 patients, who underwent elective surgeries during the incubation period of COVID-19, showed a higher risk for ICU admission and mortality [Lei 2020; Morgan 1998]. Thus, we suggest the use of a preoperative questionnaire to diagnose asymptomatic carriers and COVID-19 patients.

A retrospective analysis of elective CABG patients, during the incubation period, showed that all patients developed pneumonia after surgery with a high mortality rate [Shehata 2020; Ranney 2020]. Previous observational studies showed that mortality and complications owing to myocardial infarction was higher in patients with left ventricular dysfunction awaiting CABG or PCI [Foussas 2008; Legido-Quigley 2020]. Previous guides may help the triage of CAD patients by identifying risk. Cardiac surgery type should be reconsidered to determine the LoP. A prospective cohort analysis demonstrated that patients with severe aortic stenosis have a higher risk of mortality. Even high-performing health systems may not be resilient against this unprecedented challenge [Shehata 2020; Ranney 2020].

A fast-track discharge and minimally invasive approach to cardiac surgery or PCI procedures may decrease in-hospital nasocomial transmission of COVID-19. Transcatheter aortic valve replacement or repair also may reduce hospitalization time. Urgent cardiovascular procedures may increase the number of exposed physicians to COVID-19, if either the physicians or patients are asymptomatic carriers. COVID-19 positive healthcare professionals constituted $3.8 \%$ of patients in China [Foussas 2008; Legido-Quigley 2020]. Multicenter clinical studies comprising five confirmed and 32 suspected COVID-19 patients after emergency procedures (including cardiovascular surgery) demonstrated that guidelines of infection control can decrease nasocomial transmission in the OR [Bainbridge 2017]. None of the $41 \mathrm{HWs}$ in contact with COVID-19 patients developed an infection with COVID-19 [Tatsuishi 2018]. Therefore, preparations prior to procedures should be applied to elective cardiac surgeries to provide the safety for HWs.

\section{CONCLUSION}

The American College of Surgeons and American Society of Anesthesiologists recommended a reduction in 
new COVID-19 patients before initiation of elective surgeries [Olds 2018]. The preoperative screening for suspected patients and the professional HWs may protect both. We confirmed that the medical team was either COVID-19-negative or recovered from symptomatic COVID-19 with negative RT-PCR tests. Our consensus is LoP for elective cardiac surgery and PCI because patient comorbidity could lead to more complications during the waiting period. We also developed an appropriate scoring system to decide when to proceed or postpone. The decision to postpone an elective surgery or PCI should be made after a checklist has been completed concerning the risk of COVID-19 transmittal. Thoughtful decision-making remains the responsibility of clinics with preserving hospital health capacity, protecting both cardiac patients and healthcare providers.

Since it is not clear when the COVID-19 pandemic will end, patients who require intervention or surgery may be able to have this performed early, even it's elective. A prolonged pandemic may increase deaths, owing to CAD or its major complications, such as MACCEs. Because a number of CAD patients also have comorbid features, such as diabetes and/ or hypertension, the risk of infection and death related to COVID-19 is higher. Scientific research maintains that preparation of the operating room or Cath. Lab. prior to treatment of patients with cardiovascular diseases will help prevent the spread of COVID-19.

\section{REFERENCES}

Aguilar JB, Faust JS, Westafer LM, et al. 2020. Investigating the impact of asymptomatic carriers on COVID-19 transmission. 1;.379-94.

American College of Surgeons. 2020. COVID-19: Guidance for Triage of Non-Emergent Surgical Procedures. Available at: https://www.facs. org/covid-19/clinical-guidance/triage.

Bainbridge D, Cheng D. 2017. Current evidence on fast track cardiac recovery management. European Heart Journal Supplements. Jan $1 ; 19: 3-7$.

Bangalore, S. Atul S, Slotwiner A. 2020. ST-segment elevation in patients with COVID-19 - a case series. N Engl J Med. 382:2478-80.

Bauer A, Hausmann H, Schaarschmidt J, et al. 2018. Shed-blood-separation and cell-saver: an integral part of MiECC? Shedblood-separation and its influence on the perioperative inflammatory response during coronary revascularization with minimal invasive extracorporeal circulation systemsea randomized controlled trial. Perfusion. 136-47.

Callaway E. 2020. Time to use the p-word? Coronavirus enter dangerous new phase. Nature. 579:12.

Chan JF, Yuan S, Kok KH, et al. 2020. A familial cluster of pneumonia associated with the 2019 novel coronavirus indicating person-to-person transmission: a study of a family cluster. Lancet. 15;395:514-23.

Chen C, Zhang XR, Ju ZY, et al. 2020. Advances in the research of cytokine storm mechanism induced by Corona Virus Disease 2019 and the corresponding immunotherapies. Zhonghua shao shang Za zhi1/4 Zhonghua shaoshang zazhi1/4 Chin J Burns. 36:5.

Chen N, Zhou M, Dong X, et al. 2020. Epidemiological and clinical characteristics of 99 cases of 2019 novel coronavirus pneumonia in Wuhan, China: a descriptive study. Lancet. 15;395:507-13.
Cheng VC, Wong SC, Chen JH, et al. 2020. Escalating infection control response to the rapidly evolving epidemiology of the Coronavirus disease 2019 (COVID-19) due to SARS-CoV-2 in Hong Kong. Infection Control Hospital Epidemiology. 5:1-6.

Cheung JC, Ho LT, Cheng JV, et al. 2000. Staff safety during emergency airway management for COVID-19 in Hong Kong. Lancet Resp Med. 8(4):e19.

Chu DK, Pan Y, Cheng SM, et al. 2020. Molecular diagnosis of a novel coronavirus (2019-nCoV) causing an outbreak of pneumonia. Clinical Chemistry. 66:549-55.

Coughlin SS. 2012. Anxiety and depression: linkages with viral diseases. Public Health Rev. 34:7.

del Rio C, Malani PN. 2020. COVID-19 a new insights on a rapidly changing epidemic. Jama. Feb 28.

Dinan TG, Cryan JF. 2017. Microbes, immunity, and behavior: psychoneuroimmunology meets the microbiome. Neuropsychopharmacology. $4217 ; 89-92$.

Fang Y, Zhang H, Xie J, et al. 2020. Sensitivity of chest CT for COVID19: comparison to RT-PCR. Radiol. 19:432.

Foussas SG and Tsiaousis GZ. 2008. Revascularization treatment in patients with coronary artery disease. Hippokratia. 12(1):3-10.

Gaudriot B, Uhel F, Gregoire M, et al. 2015. Immune dysfunction after cardiac surgery with cardiopulmonary bypass: beneficial effects of maintaining mechanical ventilation. Shock. 44:228-33.

Hernandez-Palazon J, Fuentes-García D, Falcon-Arana L, et al. 2018. Assessment of preoperative anxiety in cardiac surgery patients lacking a history of anxiety: contributing factors and postoperative morbidity. J Cardiothorac Vasc Anesth. 32:236-44.

Huang C, Wang Y, Li X, et al. 2020. Clinical features of patients infected with 2019 novel coronavirus in Wuhan, China. Lancet. 15;395:497-506.

Huffmyer JL, Groves DS. 2015. Pulmonary complications of cardiopulmonary bypass. Best Practice Research Clinical Anaesthesiology. 29:163-75

Legido-Quigley H, Asgari N, Teo YY, et al. 2020. Are high-performing health systems resilient against the COVID-19 epidemic? Lancet. 95:848-50.

Lei S, Jiang F, Su W, et al. 2020. Clinical characteristics and outcomes of patients undergoing surgeries during the incubation period of COVID19 infection. Clin Med. 100:331.

Liu Y, Gayle AA, Wilder-Smith A, et al. 2020. The reproductive number of COVID-19 is higher compared to SARS coronavirus. Journal of Travel Medicine. 13;27(2).21.

Mallapaty S. 2020. Why does the coronavirus spread so easily between people? Nature. 579:183

Minamino-Muta E, Kato T, Morimoto T, et al. 2017. Causes of death in patients with severe aortic stenosis: an observational study. Sci rep. $7(1): 1-6$.

Morgan CD, Sykora K, Naylor CD. 1998. Steering Committee of the Cardiac Care Network of Ontario. Analysis of deaths while waiting for cardiac surgery among 29293 consecutive patients in Ontario, Canada. Heart. 79:345-9.

Nepogodiev D, Glasbey JC, Li E, et al. 2020. Mortality and pulmonary complications in patients undergoing surgery with perioperative SARSCoV-2 infection: an international cohort study. Lancet. 396:27-38. 
Olds A, Bashjawish B, Saadat S, et al. 2018. Minimally invasive mitral valve surgery: the new gold standard? Journal of Am College of Cardiology. 71:1978.

Poon LL, Peiris M. 2020. Emergence of a novel human coronavirus threatening human health. Nature Medicine. Feb 27:1e2.

Qiu J, Shen B, Zhao M, et al. 2020. A nationwide survey of psychological distress among Chinese people in the COVID-19 epidemic: implications and policy recommendations. Gen Psychiatr. 33(2)103-7.

Ranney ML, Griffeth V, Jha AK. 2020. Critical supply shortagesdthe need for ventilators and personal protective equipment during the Covid19 Pandemic. New Eng J Med. 30:41.

Ruan Q, Yang K, Wang W, et al. 2020. Clinical predictors of mortality due to COVID-19 based on an analysis of data of 150 patients from Wuhan, China. Intens Care Med. 3:1-3.

Shehata IM, Elhassan A, Jung JW, Urits I, Viswanath O, Kaye AD. 2020. Elective cardiac surgery during the COVID-19 pandemic: Proceed or postpone? Best Pract Res Clin Anaesthesiol. 34:643-50.

Stefanini, G. Montorfano M, Trabattoni D, Andreini D, Ferrante G, et al. 2020. ST-elevation myocardial infarction in patients with COVID-19: clinical and angiographic outcomes. Circulation. 141:2113-16.

Tatsuishi W, Nakano K, Kubota S, et al. 2018. Enhanced recovery
(Fast-Track) after cardiac and vascular surgery. In enhanced recovery after surgery. Singapore: Springer. 135-49.

Tully PJ, Baker RA. 2012. Depression, anxiety, and cardiac morbidity outcomes after coronary artery bypass surgery: a contemporary and practical review. J Geriatr Cardiol. 9:197.

Turner AJ, Hiscox JA, Hooper NM. 2004. ACE2: from vasopeptidase to SARS virus receptor. Trends in Pharmacological Sciences. 1;25(6):2910-14.

Wang D, Hu B, Hu C, et al. 2020. Clinical characteristics of 138 hospitalized patients with 2019 novel coronaviruseinfected pneumonia in Wuhan, China. Jama. 17;323:1061-9.

World Health Organization. 2005. Statement on the second meeting of the International Health Regulations (2005) Emergency Committee regarding the outbreak of novel coronavirus (2019-nCoV). Geneva, Switzerland.

World Health Organization. 2020. WHO announces COVID19 outbreak a pandemic. http://www.euro.who.int/en/health-topics/ health-emergencies/coronavirus-covid-19/ news/news/2020/3/ who-announces-covid-19-outbreak-apandemic.

Zheng YY, Ma YT, Zhang JY, et al. 2020. COVID-19 and the cardiovascular system. Nature Rev Cardiol. 5:1-2. 